|| ISSN(online): 2589-8698 || ISSN(print): 2589-868X || International Journal of Medical and Biomedical Studies

Available Online at www.ijmbs.info

PubMed (National Library of Medicine ID: 101738825)

Index Copernicus Value 2017: 40.03

\title{
A PROSPECTIVE STUDY ON THE OUTCOME OF DIFFERENT TYPES OF CATHETER USED IN HYPOSPADIAS SURGERY
}

\author{
Dr. Sudarshan Odiya ${ }^{1}$, Dr. Ashok Laddha ${ }^{1}$, Dr. Raj Kumar Mathur ${ }^{2}$, Dr. Shailendra Kumar Jharia ${ }^{3}$ \\ Associate Professor ${ }^{1}$, Professor \& Head $^{2}$, Resident ${ }^{3}$ \\ MGM, Medical College \& MY Hospital, Indore ${ }^{1,2, \& 3}$
}

Article Info: Received 27 February 2019; Accepted 23 March. 2019

Cite this article as: Odiya, Dr. S., Laddha, Dr. A., Mathur, Dr. R. K., \& Jharia, Dr. S. K. (2019). A PROSPECTIVE STUDY ON THE OUTCOME OF DIFFERENT TYPES OF CATHETER USED IN HYPOSPADIAS SURGERY. International Journal of Medical and Biomedical Studies,3(3).

DOI: https://doi.org/10.32553/ijmbs.v3i3.162

Address for Correspondence: Dr. Ashok Laddha, Associate Professor, MGM, Medical College \& MY Hospital, Indore

Conflict of interest: No conflict of interest.

\section{Abstract}

Background; Congenital penile anamoly are common in male population of paediatric age group and most common is hypospadias. Different types of catheters are being used during hypospadias repair. The objective of this study was to compared the post-operative clinico- pathological complications, patient`s compliance, effectiveness of hypospadias repair between All-silicone, Latex Foley`s, \& P.V.C stent (Feeding-tube).

Methods: Prospective study, based on inclusion and exclusion criteria with a sample size of 60 patients, study carried out in patients posted for hypospadias surgery in Department of Surgery, M.G.M Medical College and M.Y Hospital , Indore (Division of Pediatric Surgery), by a same surgeons of Paediatric Surgery Unit, M.G.M.M.C \& M.Y. Hospital INDORE, (M.P), with the use of 3 types of catheter, namely, latex foley's catheter, All-silicon foley's catheter, P.V.C-stent (feeding-tube), 20 in each group.

Results: The total number of admissions in the study period from March 2017 to March 2018 in paediatric surgery division in our hospital was -60 . Out of which, 05 was glanular hypospadias cases, 16 was coronal hypospadias cases, 11 was distal-penile hypospadias cases, 13 was mid-penile hypospadias cases. 05 was proximal-penile hypospadias cases, 10 was scrotal \&perineal hypospadias cases. Of these 60 patients, 06 - had small urethro cutaneous fistula at the site of original meatus. 01 had wound-dehiscence. 13 had need of anticholinergic/ antispasmodic Tropan. 06 required re-do surgery \& 01 had accidental dislodgement of stent.

Conclusion: All-silicon foley`s \& latex foley`s catheter having similar results. Thus, can be used in hypospadias surgery according to their availability as, CHOICE OF CATHETER in place of P.V.C stent[(feeding-tube).

Study Design: Observational Study.

Keywords: Hypospadias, All-silicon foley`s catheter, latex foley`s catheter, P.V.C stent[(feeding-tube). 


\section{Introduction:}

Infant and children have various genital abnormalities such as undescended testis, hernias, hypospadias, ambiguous genitalia etc. The most common congenital penile anomaly is hypospadias. Today various techniques and modifications are used in hypospadias repair. Different types of catheters are being used during hypospadias repair but, there were lots of confusions about use of catheter and types of catheter which gives good results in hypospadias repair. Still, today NO guidelines available to solve this issue. Some pediatric urologists prefer not to use urethral stents, to avoid inflicting pain and bladder spasm, while others favours using stents for 5-7 days, to minimize the rate of complications for the patient. Common problems associated with urinary catheters in hypospadias repair are infection, encrustation, catheter blockage, bladder spasm and trauma related to catheter insertion. In this prospective study, we aimed to compared the post-operative clinicopathological complications, patient's compliance, effectiveness of hypospadias repair between Alsilicone, Latex Foley`s, \& P.V.C stent (Feedingtube).

\section{AIMS \& OBJECTIVES;}

- To choose best catheter used in hypospadias surgery.

- To study the effectiveness \& problem of different types of catheter for improving the results of hypospadias repair.

- To study the effect of catheter, in complications of hypospadias surgery.

\section{MATERIALS \& METHODS;}

This prospective study was carried out at Department of surgery, M.G.M Medical College and M.Y Hospital Indore, after approval of Ethical Committee, for a period of 1 year.In this study total 60 patients was examined \&the patients were divided into 3 groups randomly, according to type of catheter used and each catheter group is consist of 20 patients, age $0-14$ years male .Patient undergone hypospadias surgery for first time, Parents who had gave written informed consent were included in this study .Patients along with systemic disease, on steroid therapy,redo surgery \& Parents had not given written inform consent were excluded out. Data was collected prospectively included, patient's age at operation, degree of the hypospadias, degree of associated chordee, type of surgery done, type of catheter used, length of catheter in-situ ( $5 \mathrm{~cm}$ in side the bladder ), number of days catheter in-situ, complications, need for anticholinergic / tropan, patient's compliance and cosmetic outcome.

\section{RESULTS;}

This prospective study consist on the outcome of different types of catheter used in hypospadias surgery, of all 60 paediatric patients aged 0-14 years admitted in routine \& emergency hours in Paediatric Surgery unit of Surgery department of Maharaja Yashwantrao Hospital, Indore in duration of year march 2017 to march 2018. Most of the patients with Hypospadias were from rural areas.

- $\quad$ Out of total 60 patients 45 (75\%) patients lies within 0- 5 years, 11 (18\%) patients lies within 5- 10 years, 04 (06\%) patients lies within 10- 14 years of age, 16 (27\%) patients of coronal hypospadias is the most common hypospadias diagnosed in our study, followed by $13(22 \%)$ patients of mid-penile hypospadias , 11(18\%) patients of distal-penile hypospadias, 10 (17\%) patients of scrotal-perineal hypospadias, 05 (08\%) patients of each proximal penile \&glanular hypospadias respectively.

- 45 patients lies within $\mathbf{0}$ - $\mathbf{5}$ years age group, in which 15 (33\%) patients used latex foley's catheter. 12 (26\%) patients used All-silicon foley`s catheter \& 18 (40\%) patients used P.V.C stent (feeding-tube).11 patients lies within 5- 10 years age group, in which 04 (36\%) patients used latex foley`s catheter. 06 (54\%) patients used Allsilicon foley's catheter \& 01 (09\%) patients used P.V.C stent (feeding-tube).04 patients lies within 10- 14 years age group, in which 01 (25\%) patients used latex foley's catheter. 02 (50\%) patients used All-silicon foley's catheter \& 01 
(25\%) patients used P.V.C stent (feeding-tube) urinary catheter.

- Anticholinergic drug Tropan due to spasmodic pain as a result of bladder irritation were used in 1 (6\%) patient of coronal hypospadias with latex foley's catheter. 2 patients, 1 (6\%) patient of coronal hypospadias \& other $1(7 \%)$ patient of mid-penile hypospadias with All-silicon foley's catheter having nearly significant $P$ value $(0.0050)^{[1,2,3,4]}$. \& 10 patients, $1(20 \%)$ patient of glanular hypospadias, $3(20 \%)$ patients of coronal hypospadias, $3(27 \%)$ patients of distal-penile hypospadias \& $3(23 \%)$ patients of mid-penile hypospadias with P.V.C stent (feeding-tube) urinary catheter, having significant $P$ value $(0.001)^{[5,6,7,8,9,10]}$

- Small Urethro-cutaneous fistula developed in $1(6 \%)$ patient of coronal hypospadias with All-silicon foley's catheter. 5 patients, $1(20 \%)$ patient of glanular hypospadias, 1 (6\%) patient of coronal hypospadias, 1 (9\%) patient of distalpenile hypospadias \& $2(23 \%)$ patients of midpenile hypospadias with P.V.C stent (feedingtube) urinary catheter, having significant $P$ value $(0.001)^{[1,2,3,4]}$.

- Accidently dislodgement of stent occured in 01 (09\%) patient of distal penile hypospadias with P.V.C stent (feeding-tube) urinary catheter, having significant $P$ value $(0.001)^{[5,6,7,8,9,10]}$

- Haematuria occured in 03 patients 01 (06\%) patient of coronal hypospadias, $01(09 \%)$ patient of distal penile hypospadias \& $01(07 \%)$ patient of mid-penile hypospadias with P.V.C stent (feeding-tube) urinary catheter, having significant $P$ value $(0.001){ }^{[5,6,7,8,9,10]}$.

- Wound dehiscence occured in 01(05\%) patient of distal penile hypospadias with P.V.C stent (feeding-tube) urinary catheter, having significant $P$ value (0.0039).
- Blockage of stent occured in 03 patients. 01 (06\%) patient of coronal hypospadias, 01 (09\%) patient of distal penile hypospadias \& $01(07 \%)$ patient of mid-penile hypospadias with P.V.C stent (feeding-tube) urinary catheter, having significant $P$ value $(0.001)$.

- Re-do surgery had done in 06 patients. $01(5 \%)$ patient of glanular hypospadias, $02(10 \%)$ patient of coronal hypospadias, $01(05 \%)$ patient of distal penile hypospadias \& $02(10 \%)$ patient of mid-penile hypospadias with P.V.C stent (feeding-tube) urinary catheter, having significant $P$ value $(0.001)$.

- Complication rates had been higher in P.V.C stents (feeding-tube) urethral catheterization group, in terms of urethro-cutaneous fistula, wound dehiscence, use of tropan, accidental dislodgement of stent, hematuria\& Re-do surgery.

- Very less or no complication rates in Allsilicon foley's catheter \& latex foley's catheter group. (similar complication rates in both groups).

In our study, All-silicon foley's\& latex foley`s catheter had similar results. Thus, can be used in hypospadias surgery according to their availability as, CHOICE OF CATHETER in place of P.V.C stent [(feeding-tube).

\section{TABLES \& GRAPHS.}

\section{1) AGE DISTRIBUTION.}

The mean age group of the study was 4 years with minimum age being 9 months and maximum age being 14 years. The age distribution of the disease in the study was clustered into 3 groups, mode 2, median 3 , \& mean 4 years respectively.

Table 1: Age distribution of the study population.

\begin{tabular}{|l|l|l|l|}
\hline Sr. No. & AGE. & No. OF PATIENTS. & \% AGE. \\
\hline 01$).$ & $0-5$ YEARS. & 45. & $75.00 \%$. \\
\hline 02$).$ & $5-10$ YEARS. & 11 & $18.33 \%$. \\
\hline 03). & $10-14$ YEARS. & 04 & $06.66 \%$. \\
\hline TOTAL & 60 & $100 \%$. \\
\hline
\end{tabular}


Table 2: Distribution of cases based on location of meatus.

\begin{tabular}{|l|l|l|l|}
\hline S. No. & Type of hypospadias & $\begin{array}{l}\text { No. of cases } \\
\text { (Total 60) }\end{array}$ & Percentage of cases \\
\hline 01 & Glanular hypospadias. & 05 & $08 \%$ \\
\hline 02 & Coronal hypospadias & 16 & $27 \%$ \\
\hline 03 & Distal-penile hypospadias & 11 & $18 \%$ \\
\hline 04 & Mid-penile hypospadias. & 13 & $22 \%$ \\
\hline 05 & Proximal-penile hypospadias & 05 & $08 \%$ \\
\hline 06 & Scrotal, perineal hypospadias. & 10 & $17 \%$ \\
\hline
\end{tabular}

Table 3: Distribution of Catheter used in various age group of study population.

\begin{tabular}{|l|l|l|l|l|l|}
\hline \multicolumn{2}{|l|}{ Age Groups } & $\mathbf{0 - 5}$ Years & $\mathbf{5 - 1 0}$ Years & $\mathbf{1 0 - 1 4}$ Years & Total \\
\hline \multicolumn{2}{|l|}{ Total No. of Cases } & 45 & 11 & 04 & 60 \\
\hline Foley`s Catheter & Number Of Cases & 15 & 04 & 01 & 20 \\
\cline { 2 - 6 } & Percentage & $33.33 \%$ & $36.36 \%$ & $25 \%$ & $33.33 \%$ \\
\hline \multirow{2}{*}{ All Silicon Catheter } & Number Of Cases & 12 & 06 & 02 & 20 \\
\cline { 2 - 6 } & Percentage & $26.66 \%$ & $54.54 \%$ & $50 \%$ & $33.33 \%$ \\
\hline Feeding Tube & Number of Cases & 18 & 01 & 01 & 20 \\
\cline { 2 - 6 } & Percentage & $40 \%$ & $9.09 \%$ & $25 \%$ & $33.33 \%$ \\
\hline
\end{tabular}

Table 4: Distribution of catheter based on location of meatus.

\begin{tabular}{|c|c|c|c|c|c|c|c|c|}
\hline $\begin{array}{l}\text { Sr. } \\
\text { No. }\end{array}$ & \multicolumn{2}{|l|}{ Catheter. } & $\begin{array}{l}\text { Glanular } \\
\text { hypospadias. }\end{array}$ & $\begin{array}{l}\text { Coronal } \\
\text { hypospadias. }\end{array}$ & $\begin{array}{l}\text { Distal- } \\
\text { penile- } \\
\text { hypospadias. }\end{array}$ & $\begin{array}{l}\text { Mid-penile- } \\
\text { hypospadias. }\end{array}$ & $\begin{array}{l}\text { Proximal- } \\
\text { penile- } \\
\text { hypospadias. }\end{array}$ & $\begin{array}{l}\text { Scrotal- } \\
\text { perineal } \\
\text { hypospadias. }\end{array}$ \\
\hline \multirow{2}{*}{ 01) } & \multirow{2}{*}{$\begin{array}{l}\text { Foley's } \\
\text { catheter } \\
\text { latex. }\end{array}$} & No. & 02 & 04 & 03 & 04. & 03. & 04. \\
\hline & & $\%$ & $40 \%$ & $25 \%$ & $27.27 \%$ & 30.76 & $60 \%$ & $40 \%$ \\
\hline \multirow{2}{*}{ 02) } & \multirow{2}{*}{$\begin{array}{l}\text { All- } \\
\text { silicon } \\
\text { catheter. }\end{array}$} & No. & 01. & 07. & 03. & 03. & 01. & 05. \\
\hline & & $\%$ & $20 \%$ & $43.75 \%$ & $27.27 \%$ & $23.07 \%$ & $20 \%$ & $50 \%$ \\
\hline \multirow{2}{*}{ 03) } & \multirow{2}{*}{$\begin{array}{l}\text { Feeding- } \\
\text { tube }\end{array}$} & No. & 02. & 05. & 05. & 06. & 01. & 01. \\
\hline & & $\%$ & $40 \%$ & $31.25 \%$ & $45.45 \%$ & $46.15 \%$ & $20 \%$ & $10 \%$ \\
\hline \multicolumn{3}{|c|}{ Total. } & 05. & 16. & 11. & 13. & 05. & 10. \\
\hline
\end{tabular}


Table 5: Distribution of complication of catheter based on location of meatus.

\begin{tabular}{|c|c|c|c|c|c|c|c|c|c|c|c|}
\hline \multirow[b]{3}{*}{$\begin{array}{l}\text { Sr. } \\
\text { No }\end{array}$} & \multirow{3}{*}{$\begin{array}{l}\text { Age- } \\
\text { group }\end{array}$} & \multirow{3}{*}{$\begin{array}{l}\text { Type of - } \\
\text { hypospadias }\end{array}$} & \multicolumn{9}{|l|}{ Catheter } \\
\hline & & & \multicolumn{3}{|c|}{ Foleys catheter } & \multicolumn{3}{|c|}{ All-silicon catheter } & \multicolumn{3}{|l|}{ Feeding tube } \\
\hline & & & $\begin{array}{l}\text { Complication } \\
\text { other than } \\
\text { pain. }\end{array}$ & $\begin{array}{l}\text { Redo- } \\
\text { surgery }\end{array}$ & $\begin{array}{l}\text { Use of - } \\
\text { tropan }\end{array}$ & Complication & $\begin{array}{l}\text { Redo- } \\
\text { surgery }\end{array}$ & $\begin{array}{l}\text { Use of- } \\
\text { tropan }\end{array}$ & Complication & $\begin{array}{l}\text { Redo- } \\
\text { surgery }\end{array}$ & $\begin{array}{l}\text { Use of- } \\
\text { tropan }\end{array}$ \\
\hline \multirow{6}{*}{1} & \multirow{6}{*}{$\stackrel{2}{2}$} & GLANULAR & - & - & - & - & - & - & 01 & 01 & 01 \\
\hline & & CORONAL & - & - & 01 & 01 & - & 01 & 03 & 02 & 03 \\
\hline & & DISTAL PENILE & - & - & - & - & - & - & 01 & 01 & 01 \\
\hline & & MIDDLE PENILE & - & - & - & - & - & 01 & 02 & 02 & 02 \\
\hline & & PROXIMAL PENILE & - & - & - & - & - & - & - & - & - \\
\hline & & $\begin{array}{l}\text { SCROTAL, } \\
\text { PERINEAL }\end{array}$ & - & - & - & - & - & - & - & - & 03 \\
\hline \multirow{6}{*}{2} & \multirow{6}{*}{$\frac{n}{3}$} & GLANULAR & - & - & - & - & - & - & - & - & - \\
\hline & & CORONAL & - & - & - & - & - & - & - & - & - \\
\hline & & DISTAL PENILE & . & - & . & . & - & . & . & - & . \\
\hline & & MIDDLE PENILE & 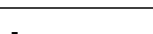 & 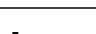 & 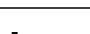 & 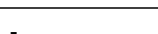 & 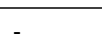 & 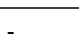 & 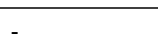 & 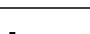 & 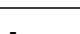 \\
\hline & & PROXIMAL PENILE & - & - & - & - & - & - & - & - & - \\
\hline & & $\begin{array}{l}\text { SCROTAL } \\
\text { PERINEAL }\end{array}$ & - & - & - & - & - & - & - & - & - \\
\hline \multirow{6}{*}{3} & \multirow{6}{*}{$\begin{array}{l}\frac{\infty}{5} \\
\frac{1}{5} \\
\frac{1}{3}\end{array}$} & GLANULAR & - & - & - & - & - & - & - & - & - \\
\hline & & CORONAL & - & - & - & - & - & - & - & - & - \\
\hline & & DISTAL PENILE & - & - & - & - & - & - & - & - & - \\
\hline & & MIDDLE PENILE & - & - & - & - & - & - & - & - & - \\
\hline & & PROXIMAL PENILE & - & - & - & - & - & - & - & - & - \\
\hline & & $\begin{array}{l}\text { SCROTAL, } \\
\text { PERINEAL }\end{array}$ & - & - & - & - & - & - & - & - & - \\
\hline \multicolumn{3}{|c|}{ Total } & - & - & 01 & 01 & - & 02 & 06 & 06 & 10 \\
\hline
\end{tabular}

Table 6: Out-come of Latex Foley`s catheter on the basis of redo-surgery, various complications, \& use of antispasmodic tropan.

\begin{tabular}{|c|c|c|c|c|c|c|c|c|c|}
\hline \multirow[b]{2}{*}{$\begin{array}{l}\text { S. } \\
\text { No. }\end{array}$} & \multirow{2}{*}{\multicolumn{2}{|c|}{$\begin{array}{l}\text { Types of } \\
\text { Hypospadias }\end{array}$}} & \multicolumn{7}{|c|}{ Complications associated with Latex Foley`s catheter } \\
\hline & & & \multirow{2}{*}{$\begin{array}{l}\text { Post } \\
\text { op } \\
\text { pain } \\
\& \\
\text { Fever } \\
02\end{array}$} & \multirow{2}{*}{$\begin{array}{l}\text { Use } \\
\text { of } \\
\text { Tropan } \\
-\end{array}$} & \multirow{2}{*}{$\begin{array}{l}\text { Fistula } \\
\text { Formation } \\
- \\
\end{array}$} & \multirow{2}{*}{$\begin{array}{l}\text { Wound } \\
\text { Dehiscence }\end{array}$} & \multirow{2}{*}{$\begin{array}{l}\text { Hematuria } \\
- \\
\end{array}$} & \multirow{2}{*}{$\begin{array}{l}\text { Accidently } \\
\text { came } \\
\text { out } \\
-\end{array}$} & \multirow{2}{*}{$\begin{array}{l}\text { Blockage } \\
-\end{array}$} \\
\hline 01 & Glanular & No. & & & & & & & \\
\hline & & $\%$ & $40 \%$ & - & - & - & - & - & - \\
\hline \multirow[t]{2}{*}{02} & \multirow[t]{2}{*}{ Coronal } & No. & 04 & 01 & - & - & - & - & - \\
\hline & & $\%$ & $25 \%$ & $06.25 \%$ & - & - & - & - & - \\
\hline \multirow[t]{2}{*}{03} & \multirow[t]{2}{*}{ Distal penile } & No. & 03 & - & - & - & - & - & - \\
\hline & & $\%$ & $27.27 \%$ & - & - & - & - & - & - \\
\hline \multirow[t]{2}{*}{04} & \multirow[t]{2}{*}{ Middle Penile } & No. & 04 & - & - & - & - & - & - \\
\hline & & $\%$ & $30.76 \%$ & - & - & - & - & - & - \\
\hline \multirow[t]{2}{*}{05} & \multirow{2}{*}{$\begin{array}{l}\text { Proximal } \\
\text { Penile }\end{array}$} & No. & 03 & - & - & - & - & - & - \\
\hline & & $\%$ & $60 \%$ & - & - & - & - & - & - \\
\hline \multirow[t]{2}{*}{06} & \multirow{2}{*}{$\begin{array}{l}\text { Scrotal- } \\
\text { Perineal }\end{array}$} & No. & 04 & - & - & - & - & - & - \\
\hline & & $\%$ & $40 \%$ & - & - & - & - & - & - \\
\hline \multicolumn{3}{|c|}{ Total } & 20 & 01 & - & - & - & - & - \\
\hline
\end{tabular}


Dr. Sudarshan Odiya et al, International Journal of Medical and Biomedical Studies (IJMBS)

Table 7: Out-come of ALL-SILICON foley`s catheter on the basis of redo-surgery, various complications, \& use of antispasmodic tropan.

\begin{tabular}{|c|c|c|c|c|c|c|c|c|c|}
\hline \multirow[b]{2}{*}{$\begin{array}{l}\text { S. } \\
\text { No. }\end{array}$} & \multirow[b]{2}{*}{$\begin{array}{l}\text { Types } \\
\text { Hypospadias }\end{array}$} & \multirow[b]{2}{*}{ of } & \multicolumn{7}{|c|}{ Complications associated with All Silicon catheter } \\
\hline & & & $\begin{array}{l}\text { Post op pain \& } \\
\text { Fever due to } \\
\text { anesthesia. }\end{array}$ & $\begin{array}{l}\text { Use } \\
\text { of } \\
\text { Tropan }\end{array}$ & $\begin{array}{l}\text { Fistula } \\
\text { Formation }\end{array}$ & $\begin{array}{l}\text { Wound } \\
\text { Dehiscence }\end{array}$ & Hematuria & $\begin{array}{l}\text { Accidently } \\
\text { came } \\
\text { out } \\
\end{array}$ & Blockage \\
\hline \multirow[t]{2}{*}{01} & \multirow[t]{2}{*}{ Glanular } & No. & 01 & - & - & - & - & - & - \\
\hline & & $\%$ & $20 \%$ & - & - & - & - & - & - \\
\hline \multirow[t]{2}{*}{02} & \multirow[t]{2}{*}{ Coronal } & No. & 07 & 01 & 01 & - & - & - & - \\
\hline & & $\%$ & $43.75 \%$ & $06.25 \%$ & $06.25 \%$ & - & - & - & - \\
\hline \multirow[t]{2}{*}{03} & \multirow[t]{2}{*}{ Distal penile } & No. & 03 & - & - & - & - & - & - \\
\hline & & $\%$ & $27.27 \%$ & - & - & - & - & - & - \\
\hline \multirow[t]{2}{*}{04} & \multirow{2}{*}{$\begin{array}{l}\text { Middle } \\
\text { Penile }\end{array}$} & No. & 03 & 01 & - & - & - & - & - \\
\hline & & $\%$ & $23.07 \%$ & $07.69 \%$ & - & - & - & - & - \\
\hline \multirow[t]{2}{*}{05} & \multirow{2}{*}{$\begin{array}{l}\text { Proximal } \\
\text { Penile }\end{array}$} & No. & 01 & - & - & - & - & - & - \\
\hline & & $\%$ & $20 \%$ & - & - & - & - & - & - \\
\hline \multirow[t]{2}{*}{06} & \multirow{2}{*}{$\begin{array}{l}\text { Scrotal- } \\
\text { Perineal }\end{array}$} & No. & 05 & - & - & - & - & - & - \\
\hline & & $\%$ & $50 \%$ & - & - & - & - & - & - \\
\hline \multicolumn{3}{|c|}{ Total } & 20 & 02 & 01 & - & - & - & - \\
\hline
\end{tabular}

Table 8: Out-come of P.V.C Stent (Feeding-tube) on the basis of redo-surgery, various complications, \& use of antispasmodic tropan.

\begin{tabular}{|c|c|c|c|c|c|c|c|c|c|}
\hline \multirow[b]{2}{*}{ S. No. } & \multirow{2}{*}{\multicolumn{2}{|c|}{$\begin{array}{l}\text { Types of } \\
\text { Hypospadias }\end{array}$}} & \multicolumn{7}{|c|}{ Complications associated with Feeding Tube catheter } \\
\hline & & & \multirow{2}{*}{$\begin{array}{l}\text { Post op pain } \\
\text { \& Fever due } \\
\text { to anesthesia } \\
02\end{array}$} & \multirow{2}{*}{$\begin{array}{l}\begin{array}{l}\text { Use Of } \\
\text { Tropan }\end{array} \\
01\end{array}$} & \multirow{2}{*}{$\begin{array}{l}\text { Fistula } \\
\text { Formation } \\
01\end{array}$} & \multirow{2}{*}{$\begin{array}{l}\text { Wound } \\
\text { Dehiscence }\end{array}$} & \multirow{2}{*}{$\begin{array}{l}\text { Hematuria } \\
- \\
\end{array}$} & \multirow{2}{*}{$\begin{array}{l}\text { Accidently } \\
\text { Came out } \\
-\end{array}$} & \multirow{2}{*}{$\begin{array}{l}\text { Blockage } \\
-\end{array}$} \\
\hline \multirow{2}{*}{01} & & No. & & & & & & & \\
\hline & Glanular & $\%$ & $40 \%$ & $20 \%$ & $20 \%$ & - & - & - & - \\
\hline \multirow{2}{*}{02} & \multirow{2}{*}{ Coronal } & No. & 05 & 03 & 01 & - & 01 & - & 01 \\
\hline & & $\%$ & $31.25 \%$ & $20 \%$ & $06.25 \%$ & - & $06.25 \%$ & - & $06.25 \%$ \\
\hline \multirow[t]{2}{*}{03} & \multirow{2}{*}{$\begin{array}{l}\text { Distal } \\
\text { penile }\end{array}$} & No. & 05 & 03 & 01 & 01 & 01 & 01 & 01 \\
\hline & & $\%$ & $45.45 \%$ & $27.27 \%$ & 09.09\% & 09.09\% & 09.09\% & 09.09\% & 09.09\% \\
\hline \multirow[t]{2}{*}{04} & \multirow{2}{*}{$\begin{array}{l}\text { Middle } \\
\text { Penile }\end{array}$} & No. & 06 & 03 & 02 & - & 01 & - & 01 \\
\hline & & $\%$ & $46.15 \%$ & $23.07 \%$ & $15.38 \%$ & - & 07.69\% & - & 07.69\% \\
\hline \multirow[t]{2}{*}{05} & \multirow{2}{*}{$\begin{array}{l}\text { Proxima } \\
\text { I Penile }\end{array}$} & No. & 01 & - & - & - & - & - & - \\
\hline & & $\%$ & $20 \%$ & - & - & - & - & - & - \\
\hline \multirow[t]{2}{*}{06} & \multirow{2}{*}{$\begin{array}{l}\text { Scrotal- } \\
\text { Perineal }\end{array}$} & No. & 01 & - & - & - & - & - & - \\
\hline & & $\%$ & $10 \%$ & - & - & - & - & - & - \\
\hline \multicolumn{3}{|l|}{ Total } & 20 & 10 & 05 & 01 & 03 & 01 & 03 \\
\hline
\end{tabular}




\section{DISCUSSION;}

- Out of total 60 patients $45(75 \%)$ patients lies within 0- 5 years, 11 (18\%) patients lies within 5- 10 years, 04 (06\%) patients lies within $10-14$ years of age, $\&$ the mean age group found is 4 years.

- 45 patients lies within $\mathbf{0 - 5}$ years age group, in which $15(33 \%)$ patients used latex foley`s catheter. $12(26 \%)$ patients used All-silicon foley`s catheter \& $18(40 \%)$ patients used P.V.C stent (feeding-tube).11 patients lies within 5- 10 years age group, in which $04(36 \%)$ patients with latex foley's catheter. 06 (54\%) patients with Allsilicon foley's catheter \& 01 (09\%) patients with P.V.C stent (feeding-tube).04 patients lies within 10- 14 years age group, in which 01 (25\%) patients used latex foley's catheter. $02(50 \%)$ patients used All-silicon foley's catheter \& 01 (25\%) patients with P.V.C stent (feeding-tube) urinary catheter.

- Anticholinergic drug Tropan due to spasmodic pain as a result of bladder irritation were used in 1 (6\%) patient of coronal hypospadias with latex foley's catheter. 2 patients, 1 (6\%) patient of coronal hypospadias \& other $1(7 \%)$ patient of mid-penile hypospadias used All-silicon foley's catheter having nearly significant $P$ value $(0.0050)^{[1,2,3,4]}$ \& \& 10 patients, 1 (20\%) patient of glanular hypospadias, $3(20 \%)$ patients of coronal hypospadias, $3(27 \%)$ patients of distal-penile hypospadias \& $3(23 \%)$ patients of mid-penile hypospadias used P.V.C stent (feeding-tube) urinary catheter, having significant $P$ value $(0.001)^{[5,6,7,8,9,10]}$

- Small Urethro-cutaneous fistula develops in $1(6 \%)$ patient of coronal hypospadias with Allsilicon foley's catheter. \& 5 patients, $1(20 \%)$ patient of glanular hypospadias, 1 (6\%) patient of coronal hypospadias, 1 (9\%) patient of distalpenile hypospadias \& $2(23 \%)$ patients of midpenile hypospadias with P.V.C stent (feedingtube) urinary catheter, having significant $P$ value $(0.001)^{[1,2,3,4]}$

- Accidently dislodgement of stent occurred in $01(09 \%)$ patient of distal penile hypospadias with P.V.C stent (feeding-tube) urinary catheter, having significant $P$ value $(0.001)^{[5,6,7,8,9,10]}$
- Haematuria occured in 03 patients 01 (06\%) patient of coronal hypospadias, $01(09 \%)$ patient of distal penile hypospadias \& $01(07 \%)$ patient of mid-penile hypospadias with P.V.C stent (feeding-tube) urinary catheter, having significant $P$ value $(0.001)$.

- Wound dehiscence occured in 01(05\%) patient of distal penile hypospadias used P.V.C stent (feeding-tube) urinary catheter, having significant $P$ value (0.0039).

- Blockage of stent occured in 03 patients. 01 (06\%) patient of coronal hypospadias, 01 (09\%) patient of distal penile hypospadias \& $01(07 \%)$ patient of mid-penile hypospadias with P.V.C stent (feeding-tube) urinary catheter, having significant $P$ value $(0.001)$.

- Re-do surgery had done in 06 patients. $01(5 \%)$ patient of glanular hypospadias, $02(10 \%)$ patient of coronal hypospadias, $01(05 \%)$ patient of distal penile hypospadias \& $02(10 \%)$ patient of mid-penile hypospadias with P.V.C stent (feeding-tube) urinary catheter, having significant $P$ value (0.001).

- Complication rates had higher in P.V.C stents (feeding-tube) urethral catheterization group, in terms of urethro-cutaneous fistula, wound dehiscence, use of tropan, accidental dislodgement of stent, hematuria\& Re-do surgery.

- Very less or no complication rates in Allsilicon foley's catheter \&latex foley's catheter group. (similar complication rates in both groups).

In our study, All-silicon foley`s \& latex foley's catheter had similar results. Thus, can be used in hypospadias surgery according to their availability as, CHOICE OF CATHETER in place of P.V.C stent[(feeding-tube).

\section{Comparision of our study with various pioneer studies;}

On comparision with Amin M. Saleh, Wesam A.\&Amr et al, Study; In which hospital stay is of 7 days, time of stent removal is 0-7 days, 15 patient $(75 \%)$, in feeding-tube urinary catheter group requires tropan, accidently dislodged stent is 04 patient (20\%), 01 patient $(5 \%)$ of latex 
foiey's catheter \& 03 patient (15\%) of feedingtube urinary catheter group develops small urethro-cutaneous fistula. 02 patient $(10 \%)$ of latex foiey's Catheter \& 05 patient (25\%) of feeding-tube urinary catheter group requires redo surgery.

SIMILAR resultsare found in our study as 1 patient (5\%) in latex foley`s, 10 patient (50\%) in feeding tubeurinary catheter group requires tropan\& having nearly significant $P$ value (0.005). accidently dislodged stent in feeding-tube urinary catheter group is 01 patient $(5 \%)$ having significant $P$ value (0.0039). 05 patient (15\%) develops small urethrocutaneous fistula, having significant $P$ value $(0.001)$ \& 06 patients $(30 \%)$ requires re-do surgery having significant $P$ value (0.001) in feeding tubeurinary catheter group.

\section{CONCLUSION}

1. $75 \%$ patients lies within $0-5$ years, $18 \%$ patients lies within 5-10 years, $06 \%$ patients lies within 10- 14 years of age range, \& The mean age group is 4 years.

2. Complication rates had higher in P.V.C Stents (feeding tube) urethral catheterization group, in terms of:

A) Urethrocutaneous fistula , significant $p$ value (0.001)

B) Wound -dehiscence, significant $p$ value (0.039)

C) Use of Tropan, nearly significant $p$ value (0.054)

D) Accidental dislodgement of stent, significant $p$ value (0.039)

E) Haematuria, significant $p$ value (0.000)

F) Re-do surgery, significant $p$ value (0.001)

3. Very less or no complication rates in AlSilicon Foleys group \& in Latex Foley`s catheter group. (similar complication rates in both groups).

In our study, All-silicon foley`s\& latex foley`s catheter had similar results. Thus, can be used in hypospadias surgery according to their availability as, CHOICE OF CATHETER in place of P.V.C stent[(feeding-tube), due to their more complications].

\section{REFERENCES}

1. Maizels M, Firlit CF. Successful hypospadias repair in infants using brief urinary diversion and watertight neourethral closure. J Urol. 145:752-754, 1986.

2. Minevich E, Pocha BR, Wacksman J, et al. Mathieu hypospadias repair : Experience in 202 patients. J Urol. 162: 2141-2142, 1999.

3. McCormack M, Homsy $Y$, LabergeY.No stent no diversion Mathieu hypospadias repair. Can J Surg.36:152-154,1993.

4. De BadiolaF,Anderson K, Gonzatez R. Hypospadias repair in an outpatient setting without proximal urinary diversion: Experience with 113 urethroplasties. J Pediatr Surg. 26:461-464, 1991.

5. Keating, MA, Duckett, JW. Operations for distal hypospadias. in: Mastery of Surgery, Urologic Surgery., Little, Brown and Company, Boston, MA; 1992:523-531. Belman, AB. Hypospadias and other urethral abnormalities. in: Clinical Pediatric Urology. vol 1.Saunders, Philadelphia, PA; 1992: 619663.

6. Winslow, BH, Devine, CJ Jr. Principles in repair of hypospadias. SeminPediatr Surg. 1996;5:41-48.Oesterling, JE, Gearhart, JP, Jeffs, RD. Urinary diversion in hypospadias surgery 1987. Urology. 1987;29:513-516.

7. Hakim, S, Merguerian, $P A$, Rabinowitz, $R$ et al, Outcome analysis of the modified Mathieu hypospadias repair: Comparison of stented and unstented repairs. J Urol. 1996;156:836-838. Mitchell, ME, Kulb, TB. Hypospadias repair without a bladder drainage catheter.J Urol.1986;135:321323.McCormack, M, Homsy, Y, Laberge, Y. "No stent, no diversion" Mathieu hypospadias repair. Can J Surg. 1993;36: 152-154.Minevich, E, Pecha, BR, Wacksman, $\mathrm{J}$ et al, Mathieu hypospadias repair: Experience in 202 patients.J Urol. 1999;162: 2141-2142.Pike, JG, Brzezinski, A, Kiruluta, $\mathrm{GH}$.

8. Use of a urethral splent in one-stage hypospadias repair. Can J Surg. 1991; 34: 507-509.Maizels, M, Firlit, CF. Successful 
hypospadias repair in infants using brief urinary diversion and watertight neourethral closure. J Urol. 1986;135:752-754.

9. De Badiola, F, Anderson, K, Gonzalez, R. Hypospadias repair in an outpatient setting without proximal urinary diversion: Experience with 113 urethroplasties. J Pediatr Surg. 1991;26:461-464.

10. Buson, H, Smiley, D, Reinberg, $Y$ et al, Distal hypospadias repair without stents: Is it better?. J Urol. 1994;151:1059-1060.

11. McCormack M, Homsy $Y$, LabergeY. No stent no diversion Mathieu hypospadias repair. Can J Surg.36:152-154,1993.

12. De BadiolaF,Anderson K, Gonzatez R. Hypospadias repair in an outpatient setting without proximal urinary diversion: Experience with 113 urethroplasties. J Pediatr Surg. 26:461-464, 1991.
13. Hakim S, Merguerian PA, Rabinowitz $R$, et al. Outcomeanalysis of the modified Mathieu hypoapdiasrepair:Comparison of stented and unstented repairs. J Urol. 156: 836-838, 1996.

14. Mitchell ME, Kulb TB. Hypospadias repair withoutbladder drainage catheter. J Urol. 153:321-323, 1986.

15. Buson H, Smiley D, Reinberg $Y$, et al. Distal hypospadiasrepair without stents: Is it better? J Urol. 151:1059-1060, 1994.

16. Pike JG, Brzezinski $A$, Kiruluta GH. Use of urethral splentin one stage hypospadias repaire. Can J Surg. 34:507-509, 1991.

17. Serdar IA, Murat MA .Urethral catheterization inhypospadias surgery: should the device enter the bladder orbe made a urethral Stent ? J Ped Surg.36:18291831, 2001. 